\title{
INCREASING THE FLEXIBILITY OF THE NAVIGATION ALGORITHM FOR INERTIAL PEDESTRIAN SYSTEMS BY CLASSIFYING MOTION TYPE WITH SUPPORT VECTOR MACHINES
}

\author{
GEORGY VOLOSNYH, GENNADY KALACH and SEKOU DIANE \\ Russian Technological University (RTU MIREA), Vernadsky Ave 78, \\ Moscow, Russia \\ E-mail: gvolosnyh@gmail.com,kalach.gennady@gmail.com,diane1990@yandex.ru, \\ www.mirea.ru
}

\begin{abstract}
This article proposes an approach to increase the flexibility of the navigation algorithm for inertial pedestrian systems by classifying the type of traffic based on the support vector machines method. The experimental studies confirm the sustainability of the proposed approach.
\end{abstract}

\section{Introduction}

At present, when satellite navigation tools have become widely available, inertial navigation systems (INS) still remain relevant where it is necessary to determine not only the coordinates of an object, but also its orientation in space; when it is impossible to receive satellite navigation signals, as well as in cases when the navigation solution should not depend on the performance of navigation satellites [1].

However, due to design peculiarities, inertial sensors tend to accumulate measurement errors over time [2]. In inertial pedestrian systems, the zero-speed correction method (ZUPT) is used to deal with this problem, which in turn implies resetting the accumulated error by the zerospeed trigger [3]. The main advantage of this approach is also its disadvantage: determining the optimal parameters for detecting zero speed is strictly tied to a specific type of movement, and in the case of different types of movement (running, walking, climbing stairs), it is not always possible to accurately detect the "zero speed" stage and therefore reset the accumulated error.

Due to the development and emergence of new approaches to classifying typical motion stages $[4,5]$, this paper proposes a way to increase the flexibility of the navigation algorithm for inertial pedestrian systems by classifying the motion type based on the support vector method (SVM) [6].

\section{Navigation algorithm for inertial pedestrian systems}

As a rule, the navigation algorithm for inertial pedestrian systems is based on the ZUPT method, which is used to detect the reference phase of a walking object and periodically correct the readings of the measuring module at the appropriate time points. Since pedestrian systems are nonlinear, the extended Kalman filter (EKF) is used as a corrector [7].

The computational cycle of the algorithm consists of two steps. In the first step, an estimate of the current navigation system errors in determining coordinates, speeds, and orientation angles is calculated. At the second step, the basic navigation solution is corrected based on the received corrections (Figure 1) [8]. 


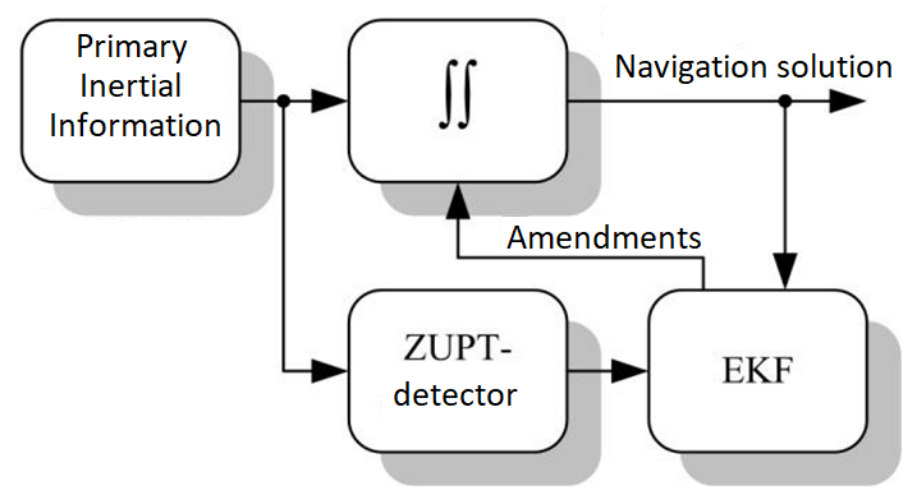

Figure 1. Block diagram of the inertial pedestrian navigation system algorithm.

During an experiment involving movement along a reference trajectory, the following real trajectory was obtained (Figure 2). The accuracy (standard deviation) was $10 \mathrm{~cm}$ (MPU-9250 was taken as a sensor).

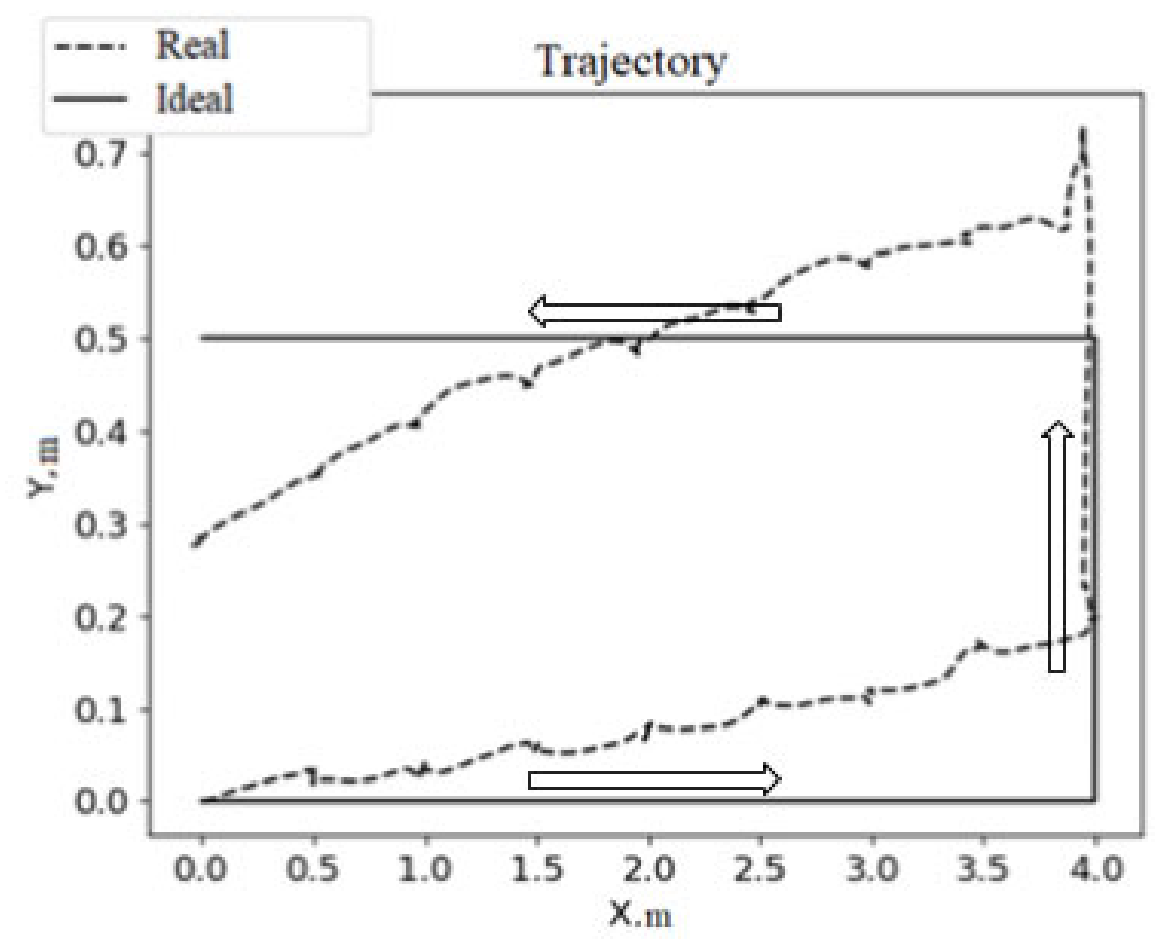

Figure 2. The resulting trajectory of the pedestrian.

\section{Adaptive zero speed detector}

To determine the optimal parameters of zero-speed detection, there are detectors [9], which are statistical tests based on various hypotheses. These detectors do not solve the problem of binding the algorithm to some a priori known type of motion, so a variant of the adaptive detector was developed, including 2 stages:

- Classifying motion type;

- Determining the optimal parameters for each of the recognized movement types.

Thus, the block diagram of the basic navigation algorithm for inertial pedestrian systems is transformed into the following (Figure 3). 


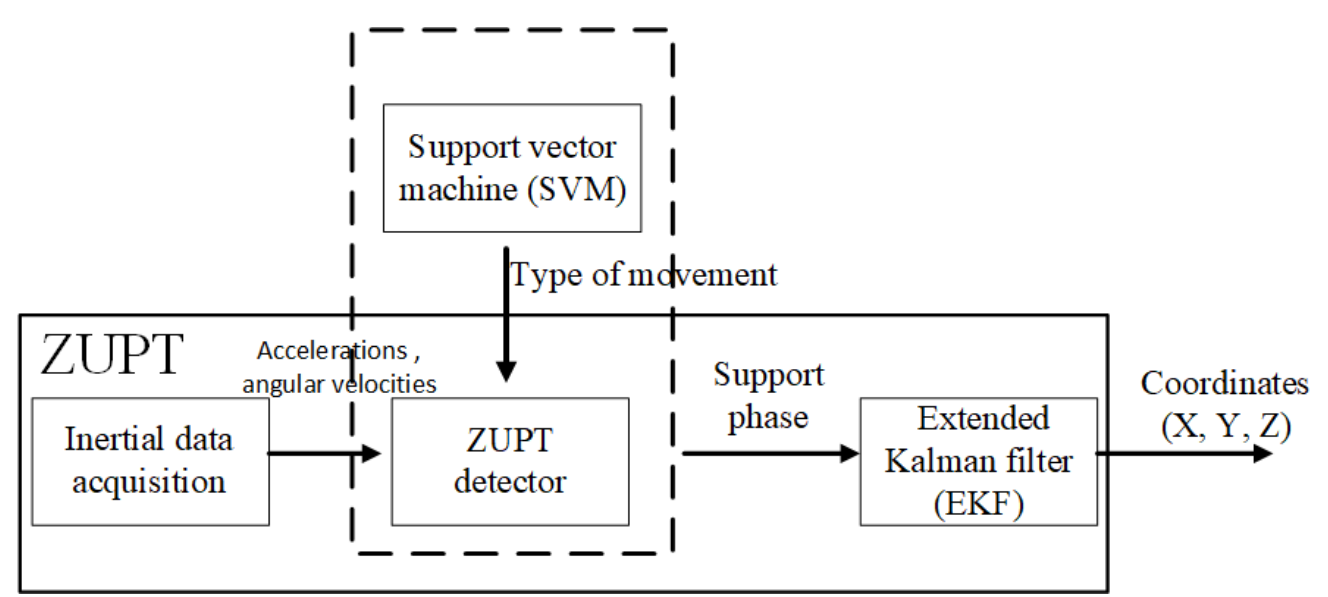

Figure 3. Updated block diagram of the inertial pedestrian navigation system algorithm.

\subsection{Classification of the movement type using the support vector machines}

To classify the type of movement, the support vector machines were used as a basis. This choice is based on previously confirmed studies found in the scientific literature. For example, authors H.-Y. Lau, K.-Y. Tong, and H. Zhu, using inertial accelerometer and gyroscope data from sensors placed on the lower leg and foot, were able to train SVM to recognize 5 types of movement. They claim a classification accuracy of $84.71 \%$ for a classifier with 5 types of movements and $100 \%$ for a classification with 2 and 3 types of movements [10].

The main idea of the support vector machines method is to translate the source vectors into a higher-dimensional space and search for a dividing hyperplane with the maximum gap in this space. In practice, the effectiveness of SVM depends on the choice of the core and parameters: C, gamma.

To train the classifier, data was taken from an accelerometer with a frequency of $100 \mathrm{~Hz}$ for 10 minutes for each type of movement: inactive (stationary) state, walking and running. The process of preparing the received data for training included the following stages:

- loading data (each class has its own dataset);

- data normalization (from 0 to 1 );

- adding labels to each class (inactive state-1, walking-2, running-3);

- creating time segments for every 2 seconds;

- finding characteristic values based on data divided by time (arithmetic mean, standard deviation, minimum and maximum values), which later serve as input parameters of the trained classifier model;

- mixing the resulting set of characteristic values;

- dividing data into training and test samples.

According to the results of cross-validation, the optimal set of parameters were: core 'linear', 'gamma' - 0.001 , and ' $\mathrm{C}$ ' -10 , where ' $\mathrm{C}$ ' is a parameter that helps to adjust the boundary between the smoothness and accuracy of the classification of objects of the training sample and 'gamma' is a parameter that determines how far each of the elements in the data set has an influence in determining the ideal line. The accuracy of the algorithm was $99 \%$, which is confirmed by the resulting matrix of inaccuracies (figure 4). 


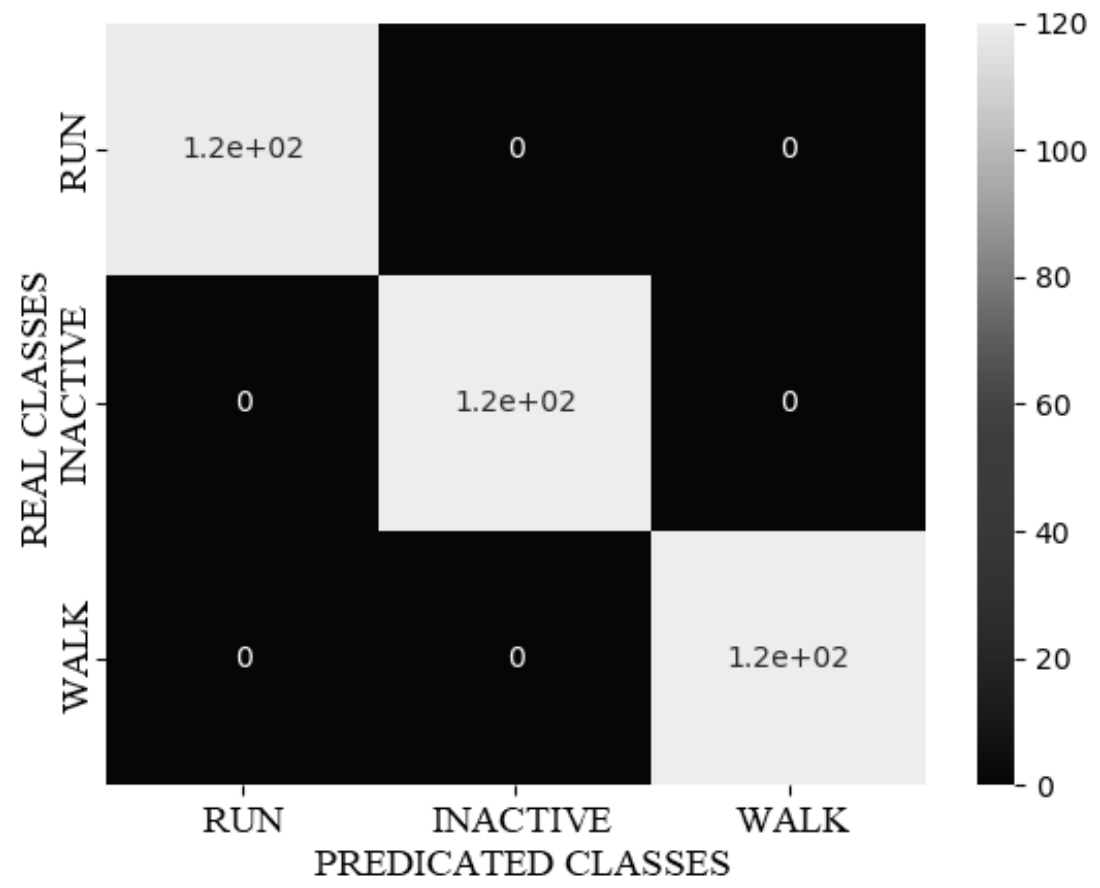

Figure 4. Confusion Matrix of trained SVM model.

\subsection{Determining the optimal threshold for each type of movement}

The Stance Hypothesis Optimal Detector (SHOE) is currently the most optimal of all zero speed detectors:

$$
T_{k}(a, \omega)=\frac{1}{W} \sum_{n=k}^{k+W-1}\left(\frac{1}{\sigma_{a}^{2}}\left\|a_{n}-g \frac{\bar{a}}{\|\bar{a}\|}\right\|^{2}+\frac{1}{\sigma_{\omega}^{2}}\left\|y_{k}^{\omega}\right\|^{2}\right) \leq \gamma
$$

$a, \omega \in R^{W \times 3}$ - measuring acceleration and angular velocity in the $\mathrm{W} ; \mathrm{W}$ - support phase detection window; $\sigma_{a}^{2}, \sigma_{\omega}^{2}$ - variance of the measurement noise of the accelerometers and gyroscopes, respectively; $g$ - gravity; $\gamma$ - detection threshold; $T_{k}$ - test statistics of the detector, $k$ - sequence number of the time interval.

The parameter $\gamma$ depends on the type of movement. This parameter can be determined by analyzing the graphs of the left side of the inequality for each of the movements. So, for example, in Figure 5, running is indicated in black solid color, and walking as a black dotted line. If you look at the values in the phases of the support, you can see that the threshold for walking (black bold line at number 2) is too low compared to the running threshold (black bold line at number 1) and is not suitable for determining zero-speed moments when running. 


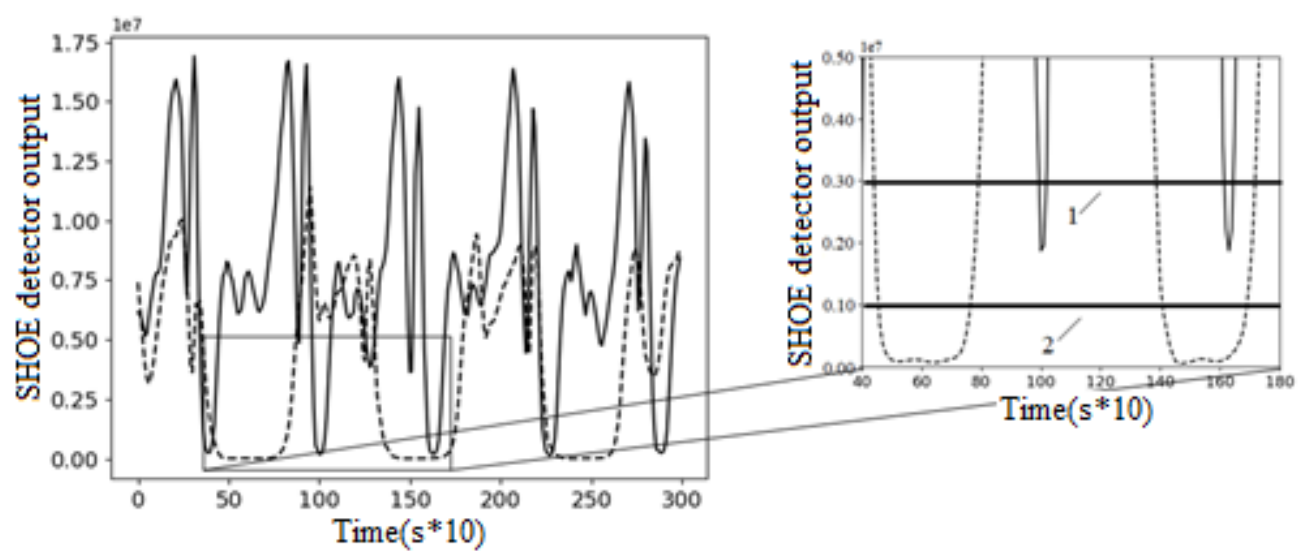

Figure 5. The graph for determining the optimal threshold for each type of movement.

Accordingly, experiments were carried out in different shoes (the sensor was located at the lower of one leg), for each of the obtained results thresholds were calculated for 2 types of movement. To obtain the optimal parameters, we calculated the arithmetic average of all experiments. Thus, $\gamma$ for walking it takes the value $1 \mathrm{e} 6$, and $\gamma$ for running-3e6.

\section{Results}

Thus, an experiment was conducted when moving along a trajectory with a change in the type of movement: from walking to running and back. The results are presented in Figure 6.

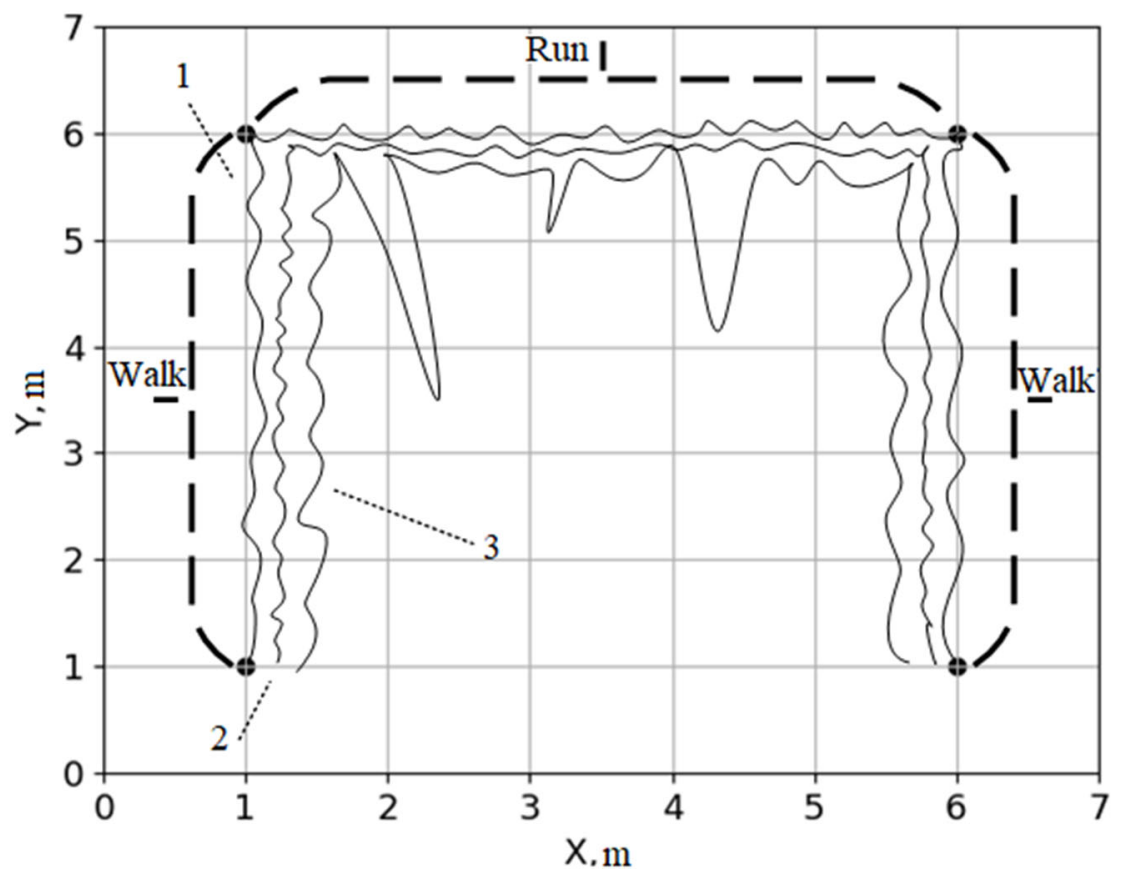

Figure 6. The graph when moving along a trajectory with a change in the type of movement.

Here, number 3 marks the resulting trajectory with a threshold for walking, number 2 with a threshold for running, and number 1 - when using an adaptive detector. As expected, the threshold for walking turned out to be too low to track the phases of support when running (occurrence of peaks in graph number 3 due to the low tracking performance), while the adaptive threshold showed the best accuracy. 


\section{Conclusion}

Based on the results obtained, it can be said that the developed software solution based on the use of the support vector machine successfully solves the problem of increasing the flexibility of the navigation algorithm for inertial pedestrian systems. This algorithm can be used for further research and implementation of its own navigation solutions.

\section{Acknowledgments}

This work is supported by the Russian Science Foundation, according to research project No. 16-19-00052 of 2020.

\section{References}

1. Vasinyova I.V., Kozlov A.V. Determination of the trajectory by the readings of inertial sensors. - MSU. Computer practical work on applied mechanics, 2015. pp. 1-18.

2. Matveev V.V., Raspopov V.Ya. Fundamentals of building of strapdown inertial navigation systems. - St.-Petersburg.: The RF State Research Center, Concern CSRI "Elektropribor", 2009. - 280 p.

3. Fischer C., Sukumar P.T., Hazas M. Tutorial: implementing a pedestrian tracker using inertial sensors. Pervasive Computing, IEEE, 2013, vol. 12, no. 2, pp. 17-27.

4. Genady G. Kalach, Alexey M. Romanov, Pavel E. Tripolskiy. Loosely coupled navigation system based on expert system using fuzzy logic. - XIX IEEE International Conference on Soft Computing and Measurements (SCM), 2016, pp. 167-169.

5. Diane S.A.K. Recognition and generation of images in neural network with hierarchical connectivity - Neurocomputers: Development, application, 2014, no. 1, pp. $47-57$

6. Fedotov D.V. On using support vector machine for solving classification problem. Siberian State Aerospace University named after academician M. F. Reshetnev. Reshetnev readings, 2013. pp. 77-79.

7. Alexey M. Romanov, Mikhail P. Romanov, Aleksandr A. Morozov, Evgeniya A. Slepynina. A Navigation System for Intelligent Mobile Robots. - IEEE Conference of Russian Young Researchers in Electrical and Electronic Engineering (EIConRus), 2019, pp. 652-656.

8. Marinushkin P.S., Bakhtina V.A., Podshivalov I.A., Stukach O.V. Issues of developing inertial pedestrian navigation systems based on MEMS sensors. - Science and education. MSTU named after N.E. Bauman. Electron. journal 2015. No. 06. pp. 157173.

9. Isaac Skog. Zero-Velocity Detection - An Algorithm Evaluation. IEEE TRANSACTIONS ON BIOMEDICAL ENGINEERING, 2010, vol. 57, no. 11, pp. 2657-2665.

10. H.-Y. Lau, K.-Y. Tong, and H. Zhu, Support vector machine for classification of walking conditions using miniature kinematic sensors, Med. Biol. Eng. Comput., vol. 46, no. 6, pp. 563-573, Jun. 2008. 